DESY 99-189

ISSN 0418-9833

MPI/PhT/99-59

hep-ph/0002058

December 1999

\title{
Virtual Sfermion Effects on Vector-Boson Pair Production at $e^{+} e^{-}$Colliders
}

\author{
A.A. Barrientos Bendezú, ${ }^{1}$ K.-P.O. Diener,${ }^{2}$ B.A. Kniehl ${ }^{1}$ \\ ${ }^{1}$ II. Institut für Theoretische Physik, Universität Hamburg, \\ Luruper Chaussee 149, 22761 Hamburg, Germany \\ ${ }^{2}$ Max-Planck-Institut für Physik (Werner-Heisenberg-Institut), \\ Föhringer Ring 6, 80805 Munich, Germany
}

\begin{abstract}
We study the quantum effects on vector-boson pair production in $e^{+} e^{-}$annihilation induced by the sleptons and squarks of the minimal supersymmetric extension of the standard model (MSSM) in the one-loop approximation. We list full analytic results, and quantitatively analyze the resulting deviation from the standard-model prediction of $e^{+} e^{-} \rightarrow W^{+} W^{-}$for the supergravity-inspired MSSM. The latter can be rendered small throughout the whole parameter space by an appropriate choice of renormalization scheme.
\end{abstract}

PACS numbers: 12.60.Jv, 13.10.+q, 14.80.Ly 
The production of $W$-boson pairs in $e^{+} e^{-}$annihilation offers a unique opportunity to probe the nonabelian gauge structure of the standard model (SM) at the tree level, which manifests itself in a distinctive cancellation between the $s^{-}, t$-, and $u$-channel scattering amplitudes. This process is being studied experimentally with high precision at the CERN Large Electron-Positron Collider (LEP2) in the centre-of-mass (CM) energy range $2 M_{W} \lesssim \sqrt{s} \lesssim 205 \mathrm{GeV}$. At the same time, the related reactions $e^{+} e^{-} \rightarrow \gamma \gamma, \gamma Z, Z Z$ are being measured there, too. With a future $e^{+} e^{-}$linear supercollider, such as JLC, NLC, or TESLA, these measurements can be extended to higher energies, way up to the $\mathrm{TeV}$ range, and rendered more precise. On the theoretical side, enormous effort has been invested into the computation of the one-loop radiative corrections to the cross sections of these processes in the SM, both for on- [1,2] and off-shell [3] vector bosons, and useful low- [4] and high-energy [5] approximations have been elaborated; for a comprehensive review, see Ref. [6].

Significant deviations of the measured cross sections from their SM predictions could signal physics beyond the SM. Since the gauge couplings of the electron are so tightly constrained by low-energy and LEP1 data, such deviations should mainly originate from the triple gauge-boson couplings (TGC's). Generic parameterizations of the so-called anomalous TGC's were introduced and applied to the processes $e^{+} e^{-} \rightarrow W^{+} W^{-}, Z Z$ in Ref. [7]. In order to explain the physical origin of anomalous TGC's, it is necessary to consider specific new-physics scenarios. From the theoretical point of view, renormalizable extensions of the SM are most satisfactory. As a rule, the deviations are then induced through loop effects of new particles, which affect not only the TGC's, but also the vector-boson propagators and the renormalizations of the parameters and wave functions of the tree-level amplitudes. Such deviations were investigated at the one-loop level in Refs. [8,9] for models with a modified lepton sector including Majorana neutrinos and were found to be generally small. In Ref. [10], the one-loop radiative corrections to the anomalous parameters $\Delta \kappa_{V}$ and $\lambda_{V}$ of the $V W^{+} W^{-}$TGC's, with $V=\gamma, Z$, were studied in the minimal supersymmetric extension of the SM (MSSM), using the pinch technique to render them gauge independent. The sfermion contributions were found to generally dominate the Higgs and gaugino contributions. This may be understood by observing that mass splittings between the up and down components of the sfermion doublets give rise to significant contributions [10] and that the sfermions come in large numbers, due to their multiplicities in flavour and colour.

In this letter, we calculate the sfermion contributions to the cross sections of $e^{+} e^{-} \rightarrow$ $V_{1} V_{2}$, with $V_{1} V_{2}=\gamma \gamma, \gamma Z, Z Z, W^{+} W^{-}$, at one loop in the MSSM. Preliminary results of this study were published in Ref. [11]. Our calculation proceeds along the lines of Ref. [9], which gives full analytic results. We use the conventions of Ref. [9] and list only those formulas which need to be substituted therein. In a way, our analysis extends Ref. [12], where the sfermion-induced radiative corrections to the processes $e^{+} e^{-} \rightarrow Z h^{0}$ and $Z \rightarrow \gamma h^{0}$, with $h^{0}$ being the lightest CP-even Higgs boson, were calculated at one loop in the MSSM. The authors of Ref. [10] did not list analytic results that could be compared with ours. In Refs. 13, 14, 15, the one-loop radiative correction to $e^{+} e^{-} \rightarrow W^{+} W^{-}$in the MSSM was considered for the full supersymmetric particle spectrum, under the simplistic 
assumption that the mass matrix of each sfermion flavour is proportional to the unit matrix, so that the two weak eigenstates are mass eigenstates with a common mass. We shall compare the sfermion loop correction of Refs. [13, 14, 15] with our result below. As we shall see later, the size of the correction is significantly affected by the sfermion mass splittings.

The Higgs sector of the MSSM is made up by two complex Higgs isodoublet of opposite hypercharge and accommodates five physical Higgs bosons: the neutral CP-even $h^{0}$ and $H^{0}$ bosons, the neutral CP-odd $A^{0}$ boson, and the charged $H^{ \pm}$-boson pair. At the tree level, it has two free parameters, which are usually taken to be the mass $m_{A}$ of the $A^{0}$ boson and the ratio $\tan \beta=v_{2} / v_{1}$ of the vacuum expectation values of the two Higgs doublets. For each of these Higgs bosons and each SM fermion and gauge boson there is a supersymmetric partner. Thus, the spectrum of states is more than doubled if one passes from the SM to the MSSM, which gives rise to a proliferation of parameters and weakens the predictive power of the theory. A canonical method to reduce the number of parameters is to embed the MSSM into a grand unified theory (GUT), e.g., a suitable supergravity (SUGRA) model, in such a way that it is recovered in the low-energy limit. The MSSM thus constrained is described by the following parameters at the GUT scale, which come in addition to $\tan \beta$ and $m_{A}$ : the universal scalar mass $m_{0}$, the universal gaugino mass $m_{1 / 2}$, the trilinear Higgs-sfermion coupling $A$, the bilinear Higgs coupling $B$, and the Higgs-higgsino mass parameter $\mu$. Notice that $m_{A}$ is then not an independent parameter anymore, but it is fixed through the renormalization group equation. The number of parameters can be further reduced by making additional assumptions. Unification of the tau and bottom Yukawa couplings at the GUT scale leads to a correlation between $m_{t}$ and $\tan \beta$. Furthermore, if the electroweak symmetry is broken radiatively, then $B$ and $\mu$ are determined up to the sign of $\mu$. Finally, it turns out that the MSSM parameters are nearly independent of the value of $A$, as long as $|A| \lesssim 500 \mathrm{GeV}$ at the GUT scale.

We now present our analytic results. We denote the four-momenta of $e^{+}, e^{-}$, and the two produced vector bosons, $V_{1}$ and $V_{2}$, by $p_{+}, p_{-}, k_{1}$, and $k_{2}$, and define the Mandelstam variables as $s=\left(p_{+}+p_{-}\right)^{2}, t=\left(p_{+}-k_{1}\right)^{2}$, and $u=\left(p_{+}-k_{2}\right)^{2}$. Neglecting the electron mass, we have $s+t+u=M_{1}^{2}+M_{2}^{2}$, where $M_{1}$ and $M_{2}$ are the masses of $V_{1}$ and $V_{2}$, respectively. In this limit, also the $s$-channel contributions due to Higgs-boson exchanges vanish. Because each of the four processes $e^{+} e^{-} \rightarrow V_{1} V_{2}$ has more than one tree-level diagram, it is convenient to introduce helicity amplitudes $\mathcal{M}^{\kappa}\left(\lambda_{1}, \lambda_{2}, s, t\right)$, where $\kappa, \lambda_{1}$, and $\lambda_{2}$ denote the helicities of $e^{-}, V_{1}$, and $V_{2}$ in the CM frame, respectively. The $e^{+}$ helicity is then $-\kappa$. The helicity amplitudes $\mathcal{M}^{\kappa}$ can be decomposed into the standard matrix elements $\mathcal{M}_{i}^{\kappa}(i=0, \ldots, 9)$ [2], which are written down in Appendix B of Ref. [9]. In addition to those for $i=0, \ldots, 3$, which already appear at the tree level, we only need $\mathcal{M}_{9}^{\kappa}$ for the present analysis.

The tree-level cross sections of $e^{+} e^{-} \rightarrow V_{1} V_{2}$ are well known and may be found in Eq. (3.1) of Ref. [9]. The sfermion-induced one-loop corrections receive contributions from diagrams containing self-energy corrections, vertex corrections, and counterterm insertions. We work in the Fermi-constant $\left(G_{F}\right)$ formulation of the electroweak on-shell renormalization scheme, which is explained in the context of Eq. (4.1) in Ref. [9]. Specif- 
ically, starting from the results in the pure on-shell renormalization scheme, which uses Sommerfeld's fine-structure constant $\alpha$ and the physical particle masses as basic parameters, we fix $\alpha=\sqrt{2} G_{F} \sin ^{2} \theta_{w} M_{W}^{2} / \pi$, where $\theta_{w}$ is the weak mixing angle, and supplement the radiative corrections with the term $-2 \Delta r$, where $\Delta r$ [16] contains those radiative corrections to the muon lifetime which the SM or its extensions introduce on top of the purely photonic corrections from within the Fermi model. The sfermion contribution to $\Delta r$ in the MSSM was examined in Ref. [12]. All the formulas listed in Section III and Appendix D of Ref. [9] carry over to the sfermion case, except for Eqs. (3.5) and (3.8), which give the transverse parts of the vector-boson vacuum polarizations $\Pi_{T}^{V_{1} V_{1}}$ and the proper vertex corrections $\delta \mathcal{M}_{V}^{\kappa}$, respectively. The relevant Feynman rules for the MSSM sfermion sector are summarized in Appendix A of Ref. [12]. For each fermion flavour $Q=U, D$, where $U=\nu_{e}, \nu_{\mu}, \nu_{\tau}, u, c, t$ and $D=e, \mu, \tau, d, s, b$, there is a corresponding sfermion flavour, denoted by a tilde. Except for the sneutrinos, which we assume to be left handed, $\tilde{Q}$ comes in two mass eigenstates $a=1,2$. The masses $M_{\tilde{Q} a}$ of the sfermions and their trilinear and quartic couplings, $\tilde{V}_{Q_{a} Q_{b}^{\prime}}^{V_{V}}$ and $\tilde{U}_{Q_{a} Q_{b}^{\prime}}^{V_{i} V_{j}}$, respectively, to the vector bosons $V_{i}=\gamma, Z, W$ are defined in Appendix A of Ref. [12]. In the absence of flavour-changing neutral currents, we have $Q=Q^{\prime}$ in $\tilde{V}_{Q_{a} Q_{b}^{\prime}}^{V_{i}}$ and $\tilde{U}_{Q_{a} Q_{b}^{\prime}}^{V_{i} V_{j}}$ if $V_{i}, V_{j}=\gamma, Z$, which explains the notation $\tilde{V}_{Q a b}^{V_{i}}$ and $\tilde{U}_{Q a b}^{V_{i} V_{j}}$ used in Ref. [12]. As in Ref. [12], we neglect the Cabibbo-Kobayashi-Maskawa mixing, so that we may write $\tilde{V}_{U a D b}^{W}$ and $\tilde{U}_{Q a b}^{W W}$. The sfermion contributions to the $\Pi_{T}^{V_{1} V_{2}}$ functions read [12]

$$
\begin{aligned}
\Pi_{T}^{V_{1} V_{2}}\left(p^{2}\right)= & \frac{1}{48 \pi^{2}} \sum_{Q, a, b} N_{\mathrm{col}}^{Q} \tilde{V}_{Q_{a} Q_{b}}^{V_{1}} \tilde{V}_{Q_{b} Q_{a}}^{V_{2}}\left\{\left[s-2\left(M_{\tilde{Q}_{a}}^{2}+M_{\tilde{Q}_{b}}^{2}\right)+\frac{\left(M_{\tilde{Q}_{a}}^{2}-M_{\tilde{Q}_{b}}^{2}\right)^{2}}{s}\right]\right. \\
& \times B_{0}\left(p, M_{\tilde{Q}_{a}}, M_{\tilde{Q}_{b}}\right)+M_{\tilde{Q}_{a}}^{2}\left(2-\frac{M_{\tilde{Q}_{a}}^{2}-M_{\tilde{Q}_{b}}^{2}}{s}\right) B_{0}\left(0, M_{\tilde{Q}_{a}}, M_{\tilde{Q}_{a}}\right) \\
& \left.+M_{\tilde{Q}_{b}}^{2}\left(2-\frac{M_{\tilde{Q}_{b}}^{2}-M_{\tilde{Q}_{a}}^{2}}{s}\right) B_{0}\left(0, M_{\tilde{Q}_{b}}, M_{\tilde{Q}_{b}}\right)+\frac{2}{3} s-\frac{\left(M_{\tilde{Q}_{a}}^{2}-M_{\tilde{Q}_{b}}^{2}\right)^{2}}{s}\right\},
\end{aligned}
$$

where $p$ is the external four-momentum, $N_{\mathrm{col}}^{Q}=1$ (3) for sleptons (squarks), and the standard two-point scalar function $B_{0}$ is defined in Eq. (C.2) of Ref. [9]. If $V_{1}=W^{-}$ and $V_{2}=W^{+}$, then $Q_{a}=U_{a}, Q_{b}=D_{b}$, and it is summed over $(U, D)$ instead of $Q$. The sfermion contribution to $\delta \mathcal{M}_{V}^{\kappa}$ is found to be

$$
\begin{aligned}
\delta \mathcal{M}_{V}^{\kappa}= & -\frac{1}{2 \pi^{2}} \sum_{B=\gamma, Z} \frac{g_{e e B}^{\kappa}}{s-M_{B}^{2}} \sum_{Q, a, b, c} N_{\text {col }}^{Q}\left(\tilde{V}_{Q_{c} Q_{b}}^{B} \tilde{V}_{Q_{b} Q_{a}}^{V_{1}} \tilde{V}_{Q_{a} Q_{c}}^{V_{2}}-\tilde{V}_{Q_{c} Q_{a}}^{V_{2}} \tilde{V}_{Q_{a} Q_{b}}^{V_{1}} \tilde{V}_{Q_{b} Q_{c}}^{B}\right) \\
& \times\left[\mathcal{M}_{1}^{\kappa}\left(C_{2}^{0}+C_{3}^{01}+C_{3}^{02}\right)+\mathcal{M}_{2}^{\kappa} C_{3}^{01}+\mathcal{M}_{3}^{\kappa} C_{3}^{02}-\mathcal{M}_{9}^{\kappa}\left(C_{2}^{12}+C_{3}^{12}+C_{3}^{21}\right)\right],
\end{aligned}
$$

where the $C$ functions are the Lorentz coefficients of the standard three-point tensor integrals defined in Eq. (C4) of Ref. [9]. In Eq. (2), we have suppressed their common 
argument $\left(k_{1},-k_{2}, M_{\tilde{Q}_{a}}, M_{\tilde{Q}_{b}}, M_{\tilde{Q}_{c}}\right)$. Deviating from Ref. [9], the electron gauge couplings appearing in Eq. (21) are defined as

$$
g_{e e \gamma}^{ \pm}=e, \quad g_{e e Z}^{+}=-\frac{e \sin \theta_{w}}{\cos \theta_{w}}, \quad g_{e e Z}^{-}=\frac{e}{\cos \theta_{w} \sin \theta_{w}}\left(\frac{1}{2}-\sin ^{2} \theta_{w}\right),
$$

where $e=\sqrt{4 \pi \alpha}$. We caution the reader that the Feynman rules used in Refs. [9, 12 differ in the sign of $\sin \theta_{w}$. Consequently, we need to multiply the expression for $\prod_{T}^{\gamma Z}$ in Eq. (1) with an extra minus sign when we insert it into the relevant formulas, Eqs. (3.6) and (D2), of Ref. [9]. Notice that Eq. (2) includes the contributions from both the direct and crossed triangle diagrams, which are proportional to $\tilde{V}_{Q_{c} Q_{b}}^{B} \tilde{V}_{Q_{b} Q_{a}}^{V_{1}} \tilde{V}_{Q_{a} Q_{c}}^{V_{2}}$ and $\tilde{V}_{Q_{c} Q_{a}}^{V_{2}} \tilde{V}_{Q_{a} Q_{b}}^{V_{1}} \tilde{V}_{Q_{b} Q_{c}}^{B}$, respectively. If $V_{1}=W^{-}$and $V_{2}=W^{+}$, then the first term contributes for $Q_{a}=U_{a}, Q_{b}=D_{b}$, and $Q_{c}=D_{c}$ and the second one for $Q_{a}=D_{a}, Q_{b}=U_{b}$, and $Q_{c}=U_{c}$.

At this point, we should compare our results with those published in Refs. 13, 14, 15]. To that end, we put $M_{\tilde{Q}_{1}}=M_{\tilde{Q}_{2}}$ and nullify the mixing angle relating the weak and mass eigenstates for each sfermion flavour $\tilde{Q}$. Then, our Eq. (1) agrees with Eqs. (C7), (D5), (E9), and (F8) in Ref. [13], up to an overall minus sign, if we eliminate the factor $1 / 2$ multiplying $T_{3 f}^{i}$ in Eq. (D5) and the sum over $i$ in Eq. (F8). As for the $\gamma W^{+} W^{-}$vertex correction, our Eq. (22) is in accordance with Eqs. (65) and (B13)-(B18) in Ref. [14] if we replace the first two appearances of $C_{36}$ in Eq. (B14) by $C_{35}$, substitute $C_{24}$ in Eq. (B18) by $C_{22}$, and include an overall minus sign in Eqs. (B17) and (B18). As for the $Z W^{+} W^{-}$ vertex correction, we find agreement with Eqs. (97) and (C48)-(C53) in Ref. [14] if we alter the overall signs of Eqs. (C52) and (C53).

Now, we explore the phenomenological implications of our results. We concentrate on the case of $e^{+} e^{-} \rightarrow W^{+} W^{-}$because, for $\sqrt{s} \gtrsim 180 \mathrm{GeV}$, it has the largest cross section of the four processes under consideration and it is the only one involving TGC's at the tree level in the SM. The SM input parameters for our numerical analysis are taken to be $G_{F}=1.16639 \cdot 10^{-5} \mathrm{GeV}^{-2}$ [17], $m_{W}=80.385 \mathrm{GeV}, m_{Z}=91.1871 \mathrm{GeV}, m_{t}=174.3 \mathrm{GeV}$ [18], and $m_{b}=4.7 \mathrm{GeV}$. We vary $\tan \beta$ and $m_{A}$ in the ranges $1<\tan \beta<35 \approx m_{t} / m_{b}$ and $100 \mathrm{GeV}<m_{A}<600 \mathrm{TeV}$, respectively. As for the GUT parameters, we choose $m_{1 / 2}=150 \mathrm{GeV}, A=0$, and $\mu<0$, and tune $m_{0}$ so as to be consistent with the desired value of $m_{A}$. All other MSSM parameters are then determined according to the SUGRA-inspired scenario as implemented in the program package SUSPECT [19]. We checked that the results obtained from the program package ISAJET 7.49 [20], where the electroweak-symmetry-breaking scale is fixed to be $Q=\sqrt{M_{\tilde{t}_{L}} M_{\tilde{t}_{R}}}$, agree with those from SUSPECT within typically $5 \%$ or less if the same scale convention is implemented in the latter. In our analysis, we adopt the SUSPECT default value $Q=M_{Z}$. We do not impose the unification of the tau and bottom Yukawa couplings at the GUT scale, which would just constrain the allowed $\tan \beta$ range without any visible effect on the results for these values of $\tan \beta$. We exclude solutions which do not comply with the present experimental lower mass bounds of the sfermions, charginos, neutralinos, and Higgs bosons [21].

In Fig. 目, the sfermion-induced correction $\delta(\theta)$ in the relationship $d \sigma / d \cos \theta=$ $(d \sigma / d \cos \theta)_{\text {Born }}[1+\delta(\theta)]$ between the one-loop-corrected and tree-level cross sections of 
$e^{+} e^{-} \rightarrow W^{+} W^{-}$is shown as a function of the scattering angle $\theta$, enclosed between the $e^{+}$and $W^{+}$three-momenta in the CM frame, for $\sqrt{s}=200,500$ and $1000 \mathrm{GeV}$ assuming $\tan \beta=10$ and $m_{A}=250 \mathrm{GeV}$. We observe that $\delta(\theta)$ has a typical size of order $0.1 \%$ or less and can be of either sign. In the backward direction, it strongly depends on the CM energy, while the energy dependence is rather feeble in the forward direction. We emphasize that the smallness of $\delta(\theta)$ is a special feature of the $G_{F}$ scheme. In the $\alpha$ scheme, the correction is given by $\delta(\theta)+2 \Delta r$ and thus shifted to negative values because we have $\Delta r \approx-0.11 \%$, as indicated in Fig. 11. Next, we study the correction $\Delta$ to the integrated cross section, defined by $\sigma=\sigma_{\text {Born }}(1+\Delta)$, for $\sqrt{s}=200 \mathrm{GeV}$. In Fig. 2, the $\tan \beta$ dependences of $\Delta$ and $\Delta r$ are shown for $m_{A}=100,250$, and $600 \mathrm{GeV}$, while, in Fig. 3, the $m_{A}$ dependences are shown for $\tan \beta=3,10$, and 30. These dependences are implicit in the sense that our formulas for $\Delta$ and $\Delta r$ do not contain $\tan \beta$ or $m_{A}$. In fact, $\Delta$ and $\Delta r$ only depend on $\tan \beta$ or $m_{A}$ via the sfermion masses and gauge couplings, the latter being affected through the mixing angles which rotate the weak eigenstates of the sfermion into their mass eigenstates. We note that the SUGRA-inspired MSSM with our choice of input parameters does not permit $\tan \beta$ and $m_{A}$ to be simultaneously small, due to the experimental selectron mass lower bound [21]. This explains why the curves for $m_{A}=100 \mathrm{GeV}$ in Fig. 2 only start at $\tan \beta \approx 11$ and those for $\tan \beta=3$ in Fig. 3 at $m_{A} \approx 240 \mathrm{GeV}$. For large $m_{A}$, the experimental $m_{h}$ lower bound [21] enforces $\tan \beta \gtrsim 3$. On the other hand, the experimental lower bounds on the chargino and neutralino masses 21] induce an upper limit on $\tan \beta$, which depends on $m_{A}$. From Fig. 2 we observe that the $\tan \beta$ dependence of $\Delta r$ for fixed $m_{A}$ is modest for intermediate values of $\tan \beta$, while $\Delta r$ increases in magnitude towards the edges of the allowed $\tan \beta$ range. The stau and tau-sneutrino contributions dominate for large $\tan \beta$ and small $m_{A}$, while the sbottom and stop contributions dominate for small $\tan \beta$ and large $m_{A}$. The contributions due to the sfermions of the first and second generations are insignificant for all values of $\tan \beta$ and $m_{A}$. It is interesting to investigate the mixings between the left- and right-handed components of the charged sfermions in the third generation. The mixing is strongest for stop, especially for small $\tan \beta$ and small $m_{A}$. For stau and sbottom, the mixings are generally feeble for large $\tan \beta$, independently of $m_{A}$. The magnitude of $\Delta r$ may reach several tenths of percent if $m_{A}$ is small to medium and $\tan \beta$ is close to its lower or upper limits. For $\tan \beta \approx 30, \Delta r$ is almost independent of $m_{A}$, while for smaller (larger) values of $\tan \beta$, the size of $\Delta r$ monotonically decreases (increases) as $m_{A}$ increases. These features are also nicely illustrated in Fig. 3. We learn from Figs. 2 and 3 that $\Delta$ is insignificant, below $0.02 \%$ in size, for all considered values of $\tan \beta$ and $m_{A}$. We stress that this happens by virtue of the $G_{F}$ scheme.

In summary, we derived analytic results for the sfermion-induced radiative corrections to the cross sections of $e^{+} e^{-} \rightarrow \gamma \gamma, \gamma Z, Z Z, W^{+} W^{-}$at one loop in the MSSM and presented a phenomenological discussion for the most interesting case, $e^{+} e^{-} \rightarrow W^{+} W^{-}$, adopting a SUGRA-inspired scenario. In the latter case, the correction can essentially be quenched by adopting the $G_{F}$ scheme, which could not be anticipated without explicit calculation. On the other hand, the sfermions are likely to generate the bulk of the MSSM correction to $e^{+} e^{-} \rightarrow W^{+} W^{-}$because of their multiplicities in flavour and 
colour. This expectation is substantiated by a study of the MSSM corrections to the $\gamma W^{+} W^{-}$and $Z W^{+} W^{-}$TGC's [10]. We conclude that significant deviations of the measured cross section of $e^{+} e^{-} \rightarrow W^{+} W^{-}$from its SM predictions will not point towards the SUGRA-inspired MSSM.

\section{Note added}

After the completion of this work, we received a preprint [22] which reports on the MSSM sfermion corrections to the cross section of $e^{+} e^{-} \rightarrow W^{+} W^{-}$in the modified minimalsubtraction scheme. The analytic results for the vector-boson vacuum polarizations and the proper vertex corrections given in Eqs. (B.1), (B.3)-(B.5), (B.8) and (B.9) of Ref. [22] agree with our Eqs. (1) and (2), respectively. The agreement was also established numerically to very high precision.

\section{Acknowledgements}

We are grateful to Ralf Hempfling for his collaboration in earlier stages of this work. We thank Jean-Loic Kneur, Gilbert Moultaka, Joannis Papavassiliou, and Jay Watson for useful comments on Ref. [10]. The work of A.A.B.B. was supported by the FriedrichEbert-Stiftung through Grant No. 219747. The II. Institut für Theoretische Physik is supported by the Bundesministerium für Bildung und Forschung under Contract No. 05 HT9GUA 3, and by the European Commission through the Research Training Network Quantum Chromodynamics and the Deep Structure of Elementary Particles under Contract No. ERBFMRXCT980194.

\section{References}

[1] M. Böhm, A. Denner, T. Sack, W. Beenakker, F. Berends, H. Kuijf, Nucl. Phys. B 304 (1988) 463; J. Fleischer, F. Jegerlehner, M. Zralek, Z. Phys. C 42 (1989) 409; J. Fleischer, K. Kolodziej, F. Jegerlehner, Phys. Rev. D 47 (1993) 830.

[2] T. Sack, Ph.D. thesis, University of Würzburg, 1987; A. Denner, T. Sack, Nucl. Phys. B 306 (1988) 221.

[3] A. Denner, T. Sack, Z. Phys. C 45 (1990) 439; D. Bardin, W. Beenakker, A. Denner, Phys. Lett. B 317 (1993) 213; K. Melnikov, O. Yakovlev, Nucl. Phys. B 471 (1996) 90; W. Beenakker, A.P. Chapovsky, F.A. Berends, Phys. Lett. B 411 (1997) 203; Nucl. Phys. B 508 (1997) 17; A. Denner, S. Dittmaier, M. Roth, Nucl. Phys. B 519 (1998) 39; Phys. Lett. B 429 (1998) 145; W. Beenakker, F.A. Berends, A.P. Chapovsky, Nucl. Phys. B 548 (1999) 3; A. Denner, S. Dittmaier, M. Roth, D. Wackeroth, Nucl. Phys. B 560 (1999) 33; Report No. BI-TP 99/45, LU-ITP 1999/020, PSI-PR-99-29, UR1591, hep-ph/9912261, December 1999; Report No. BI-TP 99/47, ER/40685/941, LU-ITP 1999/023, PSI-PR-99-34, UR-1594, hep-ph/9912447, December 1999. 
[4] S. Dittmaier, M. Böhm, A. Denner, Nucl. Phys. B 376 (1992) 29; B 391 (1993) 483 (E).

[5] W. Beenakker, A. Denner, S. Dittmaier, R. Mertig, T. Sack, Nucl. Phys. B 410 (1993) 245; W. Beenakker, A. Denner, S. Dittmaier, R. Mertig, Phys. Lett. B 317 (1993) 622 .

[6] W. Beenakker, A. Denner, Int. J. Mod. Phys. A 9 (1994) 4837.

[7] K.J.F. Gaemers, G.J. Gounaris, Z. Phys. C 1 (1979) 259; K. Hagiwara, R.D. Peccei, D. Zeppenfeld, K. Hikasa, Nucl. Phys. B 282 (1987) 253.

[8] Y. Katsuki, M. Marui, R. Najima, J. Saito, A. Sugamoto, Phys. Lett. B 354 (1995) 363.

[9] K.-P.O. Diener, B.A. Kniehl, A. Pilaftsis, Phys. Rev. D 57 (1998) 2771.

[10] A. Arhrib, J.-L. Kneur, G. Moultaka, Phys. Lett. B 376 (1996) 127.

[11] R. Hempfling, in Proceedings of the XXXIst Rencontres de Moriond: '96 Electroweak Interactions and Unified Theories, Les Arcs, France, March 16-23, 1996, ed. J. Trân Thanh Vân (Editions Frontières, Gif-sur-Yvette, 1996) p. 173; E.-A. Siemes, Diploma thesis, Technical University of Munich, 1997.

[12] R. Hempfling, B. Kniehl, Z. Phys. C 59 (1993) 263.

[13] S. Alam, Phys. Rev. D 50 (1994) 124.

[14] S. Alam, Phys. Rev. D 50 (1994) 148.

[15] S. Alam, Phys. Rev. D 50 (1994) 174.

[16] A. Sirlin, Phys. Rev. D 22 (1980) 971.

[17] C. Caso et al. (Particle Data Group), Eur. Phys. J. C 3 (1998) 1.

[18] D. Abbaneo et al. (The LEP Collaborations ALEPH, DELPHI, L3, OPAL, the LEP Electroweak Working Group and the SLD Heavy Flavour and Electroweak Groups), Report No. CERN-EP-2000-016, January 2000.

[19] A. Djouadi, J.-L. Kneur, G. Moultaka, Report No. PM/98-27, GDR-S-017 (1998).

[20] H. Baer, F.E. Paige, S.D. Protopopescu, X. Tata, Report No. BNL-HET-99-43, hepph/0001086, December 1999.

[21] V. Ruhlmann-Kleider, Report No. hep-ex/0001061, January 2000, to be published in the Proceedings of 19th International Symposium on Lepton and Photon Interactions at High Energies (LP 99), Stanford, California, 9-14 August 1999. 
[22] S. Alam, K. Hagiwara, S. Kanemura, R. Szalapski, Y. Umeda, Report No. KEK-TH670, KA-TP-27-1999, UR-1601, DESY 99-201, hep-ph/0002066, February 2000. 


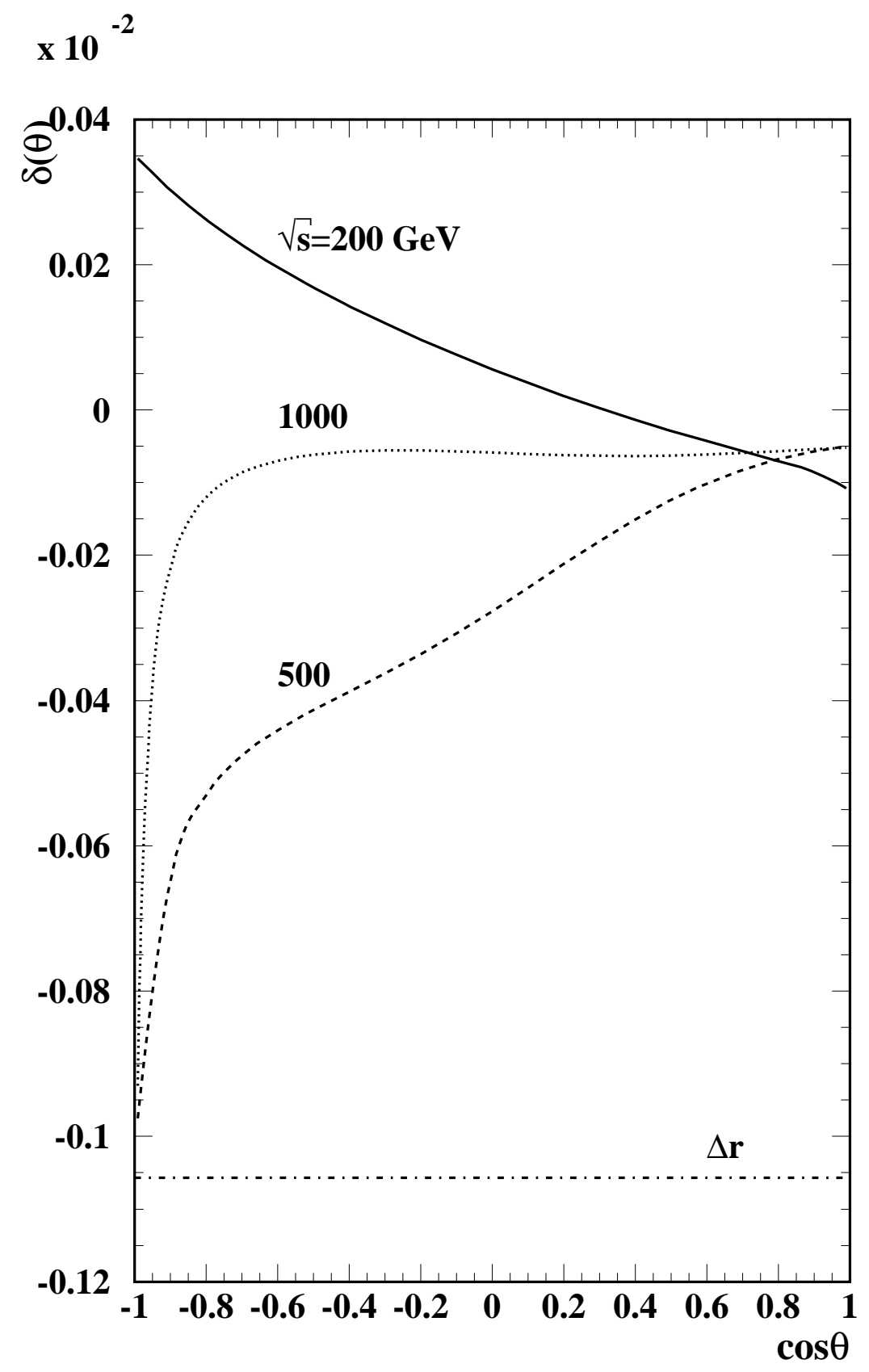

Figure 1: Relative correction $\delta(\theta)$ to the differential cross section $d \sigma / d \cos \theta$ of $e^{+} e^{-} \rightarrow$ $W^{+} W^{-}$, for $\sqrt{s}=200,500$, and $1000 \mathrm{GeV}$, and contribution to $\Delta r$ due to the sfermions in the SUGRA-inspired MSSM with $\tan \beta=10$ and $m_{A}=250 \mathrm{GeV}$, as functions of $\cos \theta$. 


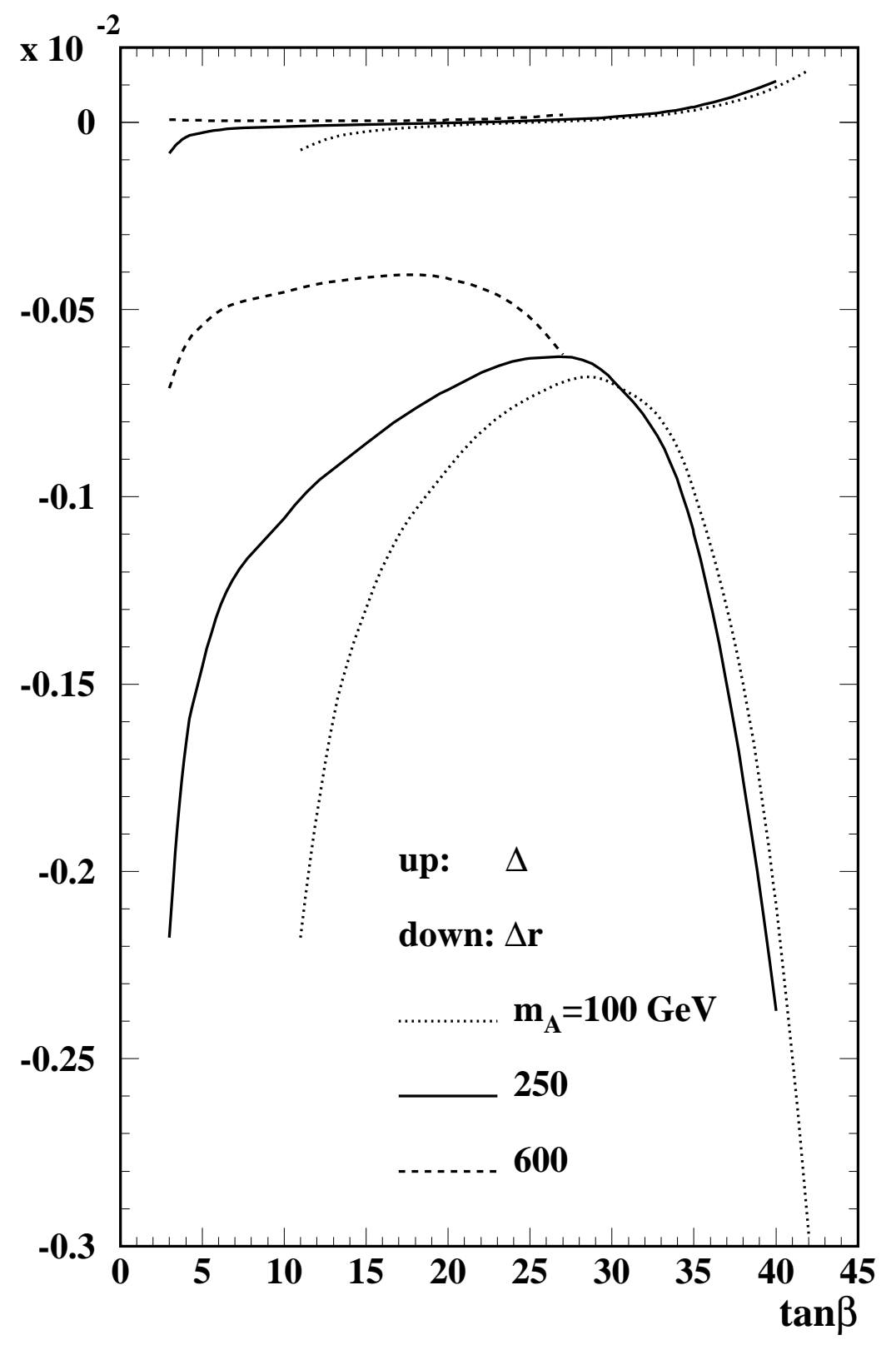

Figure 2: Relative correction $\Delta$ to the total cross section $\sigma$ of $e^{+} e^{-} \rightarrow W^{+} W^{-}$, for $\sqrt{s}=200 \mathrm{GeV}$, and contribution to $\Delta r$ due to the sfermions in the SUGRA-inspired MSSM with $m_{A}=100,250$, and $600 \mathrm{GeV}$, as functions of $\tan \beta$. 


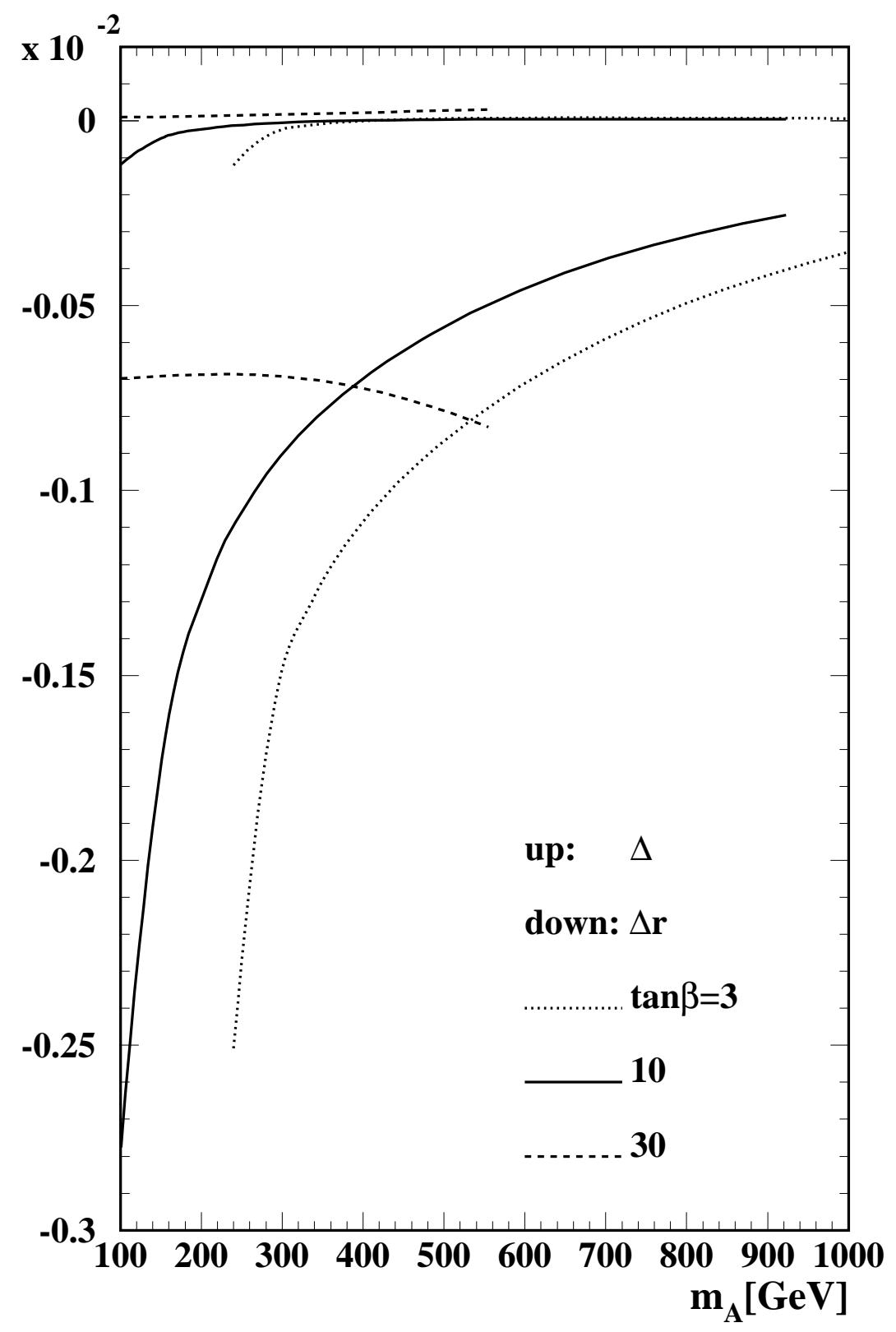

Figure 3: Relative correction $\Delta$ to the total cross section $\sigma$ of $e^{+} e^{-} \rightarrow W^{+} W^{-}$, for $\sqrt{s}=200 \mathrm{GeV}$, and contribution to $\Delta r$ due to the sfermions in the SUGRA-inspired MSSM with $\tan \beta=3,10$, and 30 , as functions of $m_{A}$. 